\title{
Influencia del Recubrimiento de las Fibras de Vidrio sobre la Efectividad de la Reacción de Copolimerización Propileno-Vidrio
}

\author{
Mariana Etcheverry, María L. Ferreira, Numa J. Capiati y Silvia E. Barbosa \\ Planta Piloto de Ingeniería Química, Universidad Nacional del Sur (PLAPIQUI-UNS-CONICET), \\ Camino La Carrindanga km 7, 8000 Bahía Blanca-Argentina \\ (e-mail: metcheverry@plapiqui.edu.ar)
}

Recibido Dic. 15, 2010; Aceptado Feb. 08, 2011; Versión Final Recibida Feb. 23, 2011

\section{Resumen}

Se ha analizado la influencia de recubrimientos de fibras sobre la efectividad de la reacción de copolimerización de propileno-vidrio. Las propiedades mecánicas de los materiales compuestos reforzados con fibras dependen de la transferencia efectiva de carga fibra-matriz, la cual es posible si la adhesión entre ambas es alta. Una alternativa de mejoramiento de la adhesión es el establecimiento de vínculos de naturaleza química matriz-fibra como la polimerización in situ. El estudio se lleva a cabo sobre compuestos sándwich de polipropileno y redes de fibra de vidrio, con y sin recubrimiento de acrilato. Se analiza adhesión a través de su influencia en las propiedades mecánicas de los compuestos. La reacción de copolimerización es muy efectiva cuando se lleva a cabo sobre fibras sin recubrimientos, haciendo la propuesta tecnológicamente más atractiva.

Palabras clave: materiales compuestos, polimerización in situ, recubrimiento de fibras, adhesión

\section{Glass-fiber Coating Influence on the Effectiveness of Propylene-Glass Copolymerization Reaction}

\begin{abstract}
The influence of fiber coatings on the effectiveness of the propylene-glass copolymerization reaction has been analyzed. The mechanical properties of fiber reinforced composites depend on the effective stress transfer fiber-matrix, which is possible if the adhesion between them is strong. An alternative method to improve the adhesion is the establishment of chemical links between matrix-fiber such as in situ polymerization. The study was carried out on sandwich composite of polypropylene and fiber glass, with and without acrylic coating. Adhesion is analyzed through its influence on the mechanical properties of the composites. The copolymerization reaction acts as an effective bonding agent, when it is carried out on uncoated fibers, making this proposal technological attractive.
\end{abstract}

Keywords: composites, in-situ polymerization, fiber coating, adhesion 


\section{INTRODUCCIÓN}

Las propiedades mecánicas de los materiales compuestos reforzadas con fibras dependen de la transferencia de carga efectiva fibra-matriz. Esto hace que la adhesión entre ambas fases sea un factor fundamental para obtener materiales de alta performance dado que cuando la adhesión no es eficaz, las fibras no funcionan como un material de refuerzo efectivo (Mallick, 2003). Se han llevado a cabo muchos esfuerzos para incrementarla, pero particularmente, una alternativa promisoria para su mejoramiento es el establecimiento de vínculos de naturaleza química entre la matriz y la fibra. En nuestro grupo se ha desarrollado un método para incrementar la adhesión entre polipropileno (PP) y fibras de vidrio (FV), injertando propileno sobre la superficie de las fibras (Etcheverry et al., 2007). Los resultados obtenidos muestran que la adhesión mejora notablemente y mucho más que con otros sistemas estudiados en la literatura (Bledzki et al., 1999; DiBenedetto, 2001; Feller y Grohens, 2004; Monte, 2005; Arbelaiz et al., 2005; Xantos, 2005).

Las FV se comercializan recubiertas con distintos agentes. El objetivo de este trabajo es analizar la influencia del recubrimiento sobre la efectividad de la reacción de copolimerización de propileno-vidrio y consecuentemente sobre la adhesión en compuestos PP/FV. La reacción se lleva a cabo sobre tejidos de fibras en forma de redes, una sin recubrimiento y otras recubiertas con acrilatos. La ocurrencia de la misma se estudia comparativamente utilizando microscopía electrónica de barrido con análisis de rayos X dispersivo (SEM-EDX), en línea. La variación de la adhesión se analiza midiendo propiedades mecánicas a tracción sobre compuestos sándwich PP/FV/PP.

\section{EXPERIMENTAL}

\section{Materiales}

Como matriz de los compuestos se utilizó una película comercial de un copolímero propilenoetileno (Moplen RP348T, Basell) provisto por el Lavorazioni Materie Plastiche (Vicenza, Italia). El copolímero tiene un contenido de etileno menor al 4\%. El espesor nominal de la película es de 78 $\mu \mathrm{m}$. El peso molecular del mismo, medido por cromatografía por permeación de geles (GPC), es $M w=198900 \mathrm{~g} / \mathrm{mol}$ y su polidispersión 2,71 . En tanto que se utilizaron dos tejidos de fibra de vidrio, uno con recubrimiento de acrilato comercial y trama de $250 \mathrm{~g} / \mathrm{m}^{2}$, denominado $R F$, y otro con fibra de vidrio sin recubrimiento y trama de $900 \mathrm{~g} / \mathrm{m}^{2}$, denominado RG. El diámetro nominal de los monofilamentos que integran las redes es $25 \mu \mathrm{m}$.

\section{Reacción de copolimerización propileno-vidrio}

Sobre las redes de fibra se llevó a cabo la reacción que se describe en (Etcheverry et al., 2007). Básicamente, el primer paso es la incorporación de metilaluminoxano (MAO) a la superficie a través de los grupos oxidrilo de las fibras de vidrio. Los grupos metilo remanentes del MAO reaccionan con el hidrógeno activo de la hidroxi-a-olefina (9-decen-1-ol), generando un macromonómero con desprendimiento de metano y la fijación al vidrio de grupos vinilo terminal. Este macromonómero se copolimeriza con propileno en presencia del catalizador metalocénico $\left(\mathrm{EtInd}_{2} \mathrm{ZrCl}_{2}\right)$ activado por MAO que se adiciona al medio de reacción. La reacción se lleva a cabo durante 45 minutos a $70^{\circ} \mathrm{C}$ hasta consumo total de la hidroxi-a-olefina. Por último, para precipitar el polímero y desactivar/descomponer el catalizador se agrega una mezcla de etanol/HCl.

\section{Caracterización morfológica}

La morfología final de las muestras, así también como una evidencia indirecta de la ocurrencia de la reacción de polimerización in-situ, se obtuvo analizando comparativamente las mismas por SEM/EDX. 


\section{Pruebas mecánicas}

Se llevaron a cabo ensayos de tracción en un espectrómetro mecánico Instron 3369 a temperatura ambiente, con una velocidad de $5 \mathrm{~mm} / \mathrm{min}$ utilizando una celda de carga de $1 \mathrm{kN}$. Los compuestos se prepararon colocando la red, RF o RG, entre las películas de PP (tipo sándwich PP/FV/PP), se fundieron en prensa hidráulica a $165{ }^{\circ} \mathrm{C}$ durante 3 minutos y se dejaron enfriar a temperatura ambiente. La forma y dimensión de las muestras para los ensayos mecánicos se muestra en la Figura 1. Con cada red se prepararon tres tipos de compuestos, dependiendo del tratamiento efectuado a la red. En la Tabla 1 se listan todos los compuestos preparados. Notar que se prepararon compuestos con redes tratadas sólo con MAO a fin de analizar posibles variaciones de propiedades mecánicas debidas a la influencia del óxido de aluminio remanente sobre el vidrio.

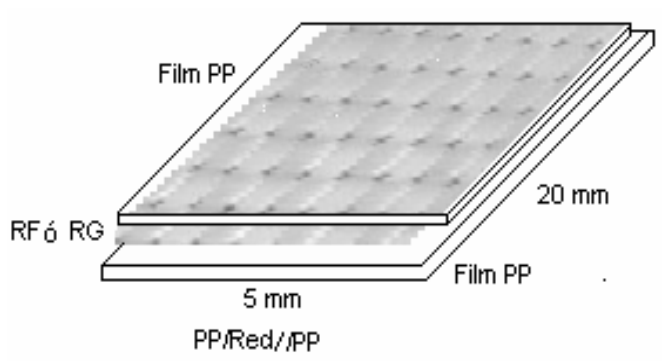

Fig.1. Formas y dimensiones de los compuestos sándwich para los ensayos mecánicos

Tabla 1. Denominación de las muestras sometidas a ensayos mecánicos

\begin{tabular}{|c|c|c|}
\hline Red sin tratamiento previo & $\begin{array}{c}\text { Red tratada con MAO } \\
\text { (RF-MAO y RG-MAO) }\end{array}$ & $\begin{array}{c}\text { Red copolimerizada } \\
\text { (RF-COP y RG-COP) }\end{array}$ \\
\hline PP/RF/PP & PP/RF-MAO/PP & PP/RF-COP/PP \\
\hline PP/RG/PP & PP/RG-MAO/PP & PP/RG-COP/PP \\
\hline
\end{tabular}

\section{RESULTADOS Y DISCUSIÓN}

La figura 2 muestra las micrografías SEM, con su correspondiente espectro EDX, de la RF y RG sin polimerizar. En el espectro EDX de la RF aparece un pico de $C$ relativamente alto debido a que la RF está revestida de un copolímero de ácido acrílico resistente a sustancias alcalinas. Este recubrimiento cambia las condiciones superficiales de las fibras a ser copolimerizadas. Por otra parte, el espectro de la RG presenta sólo los picos característicos del vidrio correspondientes a $\mathrm{Si}$, Al, Ca y O.

La figura 3 muestra las redes luego de ser sometidas a la reacción de copolimerización. Ambos tejidos de fibras presentan polímero en su superficie. En el caso de la RF, el pico de C del espectro EDX de las muestras polimerizadas es mucho mayor que el de las muestras sin polimerizar (Figs. 2a y 3a). La relación de picos $\mathrm{C} / \mathrm{Si}$ se incrementa notablemente, a punto tal que en la muestra RF-COP desaparece el pico de Si evidenciando así un drástico aumento del pico de C. Con respecto a la muestra RG-COP se observa la aparición de un gran pico de $C$ ausente en el espectro de partida (Figs. $2 \mathrm{~b}$ y $3 \mathrm{~b}$ ). Estos resultados concuerdan con el bajo grado de cubrimiento obtenido en las muestras RF respecto de la RG evidenciado en las micrografías de la Fig. 3 tomadas a igual magnificación. 

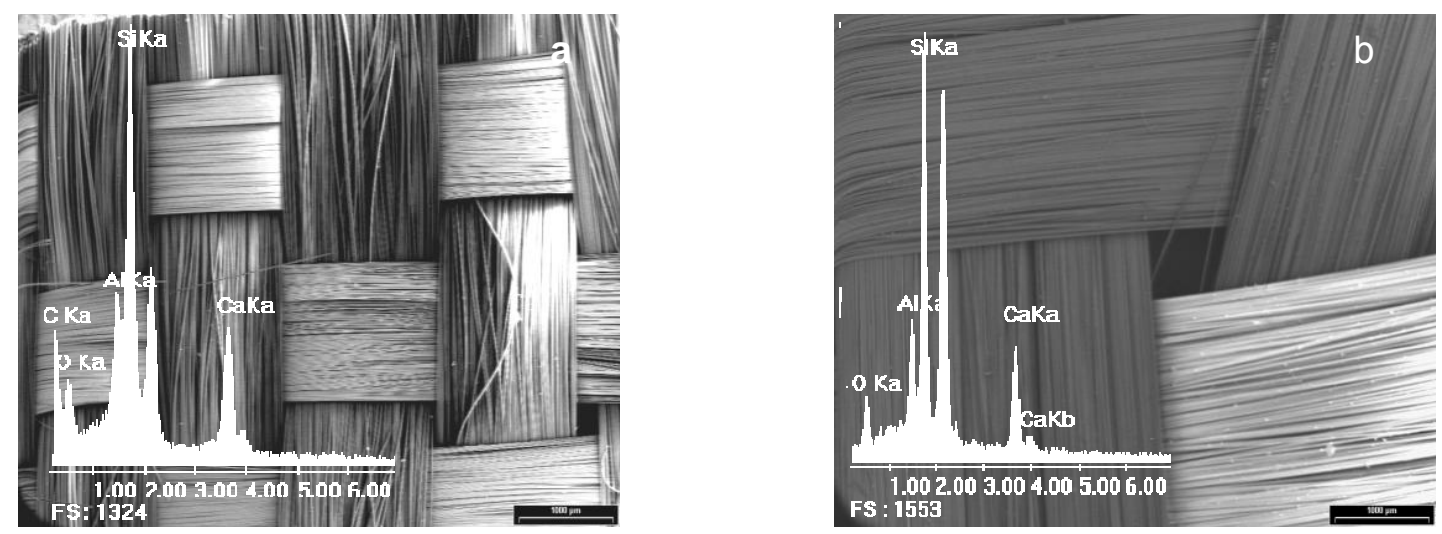

Fig. 2. Micrografías SEM (20 x), con su correspondiente espectro EDX, de a) red fina y b) red gruesa
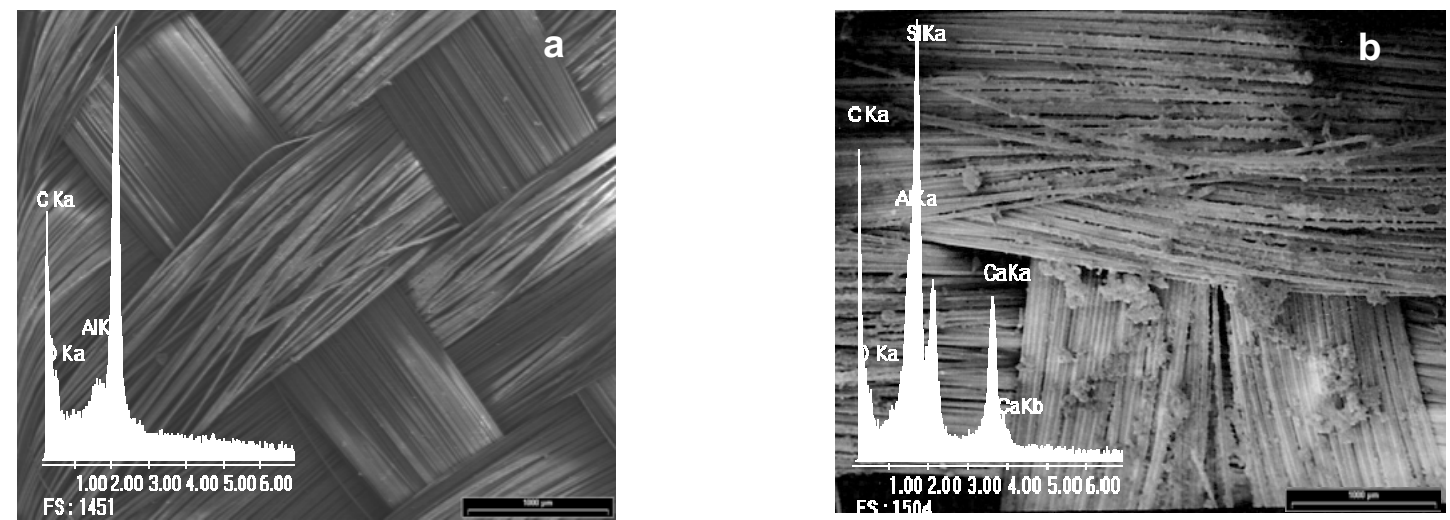

Fig. 3. Micrografías SEM (20 x), de a) RF-COP sobre recubrimiento de acrilatos b) RG-COP con sus correspondientes espectros EDX

El comportamiento mecánico a tracción de todos los materiales compuestos preparados se presenta en la figura 4 en tanto que las propiedades se resumen en la tabla 2. Cómo es esperable, para cada grupo de compuestos con una misma red base, el módulo elástico no presenta variaciones considerables dado que, la concentración de fibras es análoga. Sin embargo este parámetro se incrementa en los compuestos que utilizan red gruesa porque contienen mayor cantidad de fibras. Cabe destacar que la mayor contribución teórica de las fibras al módulo elástico se corresponde con la regla de las mezclas (fibras alineadas en la dirección del esfuerzo). En tanto que la menor contribución posible viene dada cuando todas las fibras están perpendiculares al esfuerzo. En el caso de las redes, el módulo elástico será un valor comprendido entre los anteriores (Nielsen y Landel, 1994). Fijada la orientación, en ambos casos el parámetro más importante es la concentración de fibras.

La formas de las curvas de los compuestos RF (Fig. 4a) es típica de los laminados en dos direcciones cruzadas "cross-ply laminate", dónde a una elongación de aproximadamente $3 \%$ se rompen las fibras en una dirección y continúan resistiendo las otras (Nielsen y Landel, 1994). Por otra parte los compuestos tienen un comportamiento frágil no presentan fluencia, consiguiéndose la mayor resistencia mecánica y elongación a rotura en los materiales cuya fibras no fueron modificadas (PP/RF/PP). Es notable que los compuestos PP/RF-MAO/PP y PP/RF-COP/PP tienen comportamientos muy similares y propiedades menores a PP/RF/PP. Esto podría atribuirse a que el tratamiento con MAO induce fallas en el recubrimiento acrílico y afecta la resistencia de las fibras. El aumento notable en la elongación en los compuestos PP/RF/PP no es a causa de una mayor compatibilidad con el PP sino a causa de la propia resistencia de la red con su recubrimiento, lo que es evidente comparándolo con la curva de RF sin PP. 
El comportamiento mecánico a tracción de los compuestos preparados con la RG es completamente distinto (Fig. 4 b) a los preparados con la RF, en este caso las curvas presentan un máximo que determina la resistencia mecánica que a su vez es distinta de la tensión de rotura. En los compuestos PP/RG/PP y PP/RG-MAO/PP la resistencia mecánica se alcanza a una deformación de entre 4 y $5 \%$ mientras que para el compuesto PP/RG-COP/PP, el valor se duplica, aprox. $10 \%$. Esto está directamente relacionado con la forma de las curvas donde es claro que para las dos primeras el material se rompe casi drásticamente a partir de ese punto, en el cual las fibras se rompen masivamente $(3 \%)$ y comienza a resistir el polímero que no está bien adherido a la fibra. Sin embargo, la gran diferencia se encuentra en el valor de la resistencia mecánica. Los compuestos con las fibras copolimerizadas triplican el valor de las otras muestras, este resultado puede entenderse teniendo en cuenta que en las muestras PP/RG/PP y PP/RGMAO/PP, la película de PP literalmente se despega del tejido dado que no penetra en el mismo y por ende la adhesión es mala y sólo superficial. Esto hace que a medida que la prueba mecánica se desarrolla, el film se vaya despegando, la resistencia es similar a la del PP puro y la "rotura" sea por despegado total del mismo. Esto no sucede cuando las fibras están copolimerizadas dado que se incrementan no solo la adhesión sino también la penetrabilidad del polipropileno en la red. Los valores de elongación a rotura se incrementan levemente sin embargo el compuesto PP/RGCOP/PP es mucho más tenaz dado que requiere mucha mayor energía para romperse (área bajo la curva) (Etcheverry et al., 2008).

Las fibras copolimerizadas aumentan la adhesión y por ende las propiedades mecánicas (Etcheverry et al., 2008). De los resultados anteriores surge que la reacción de copolimerización de propileno, tiene alta efectividad cuando se lleva a cabo sobre fibras sin recubrimientos, es decir sobre los $\mathrm{OH}$ propios del vidrio y no es efectiva cuando las fibras están recubiertas con acrilatos. Cuando el copolímero está químicamente adherido y su longitud es suficiente para producir entrelazamientos efectivos con la matriz, la fractura se produce dentro de esta con un mayor impedimento del despegado de las fibras. Por otra parte, la no variación del comportamiento mecánico de los compuestos RF preparados con fibras polimerizadas y tratadas sólo con MAO y su menor valor respecto del de los compuestos preparados con fibras no tratadas evidencia que los entrelazamientos no determinan el comportamiento mecánico.
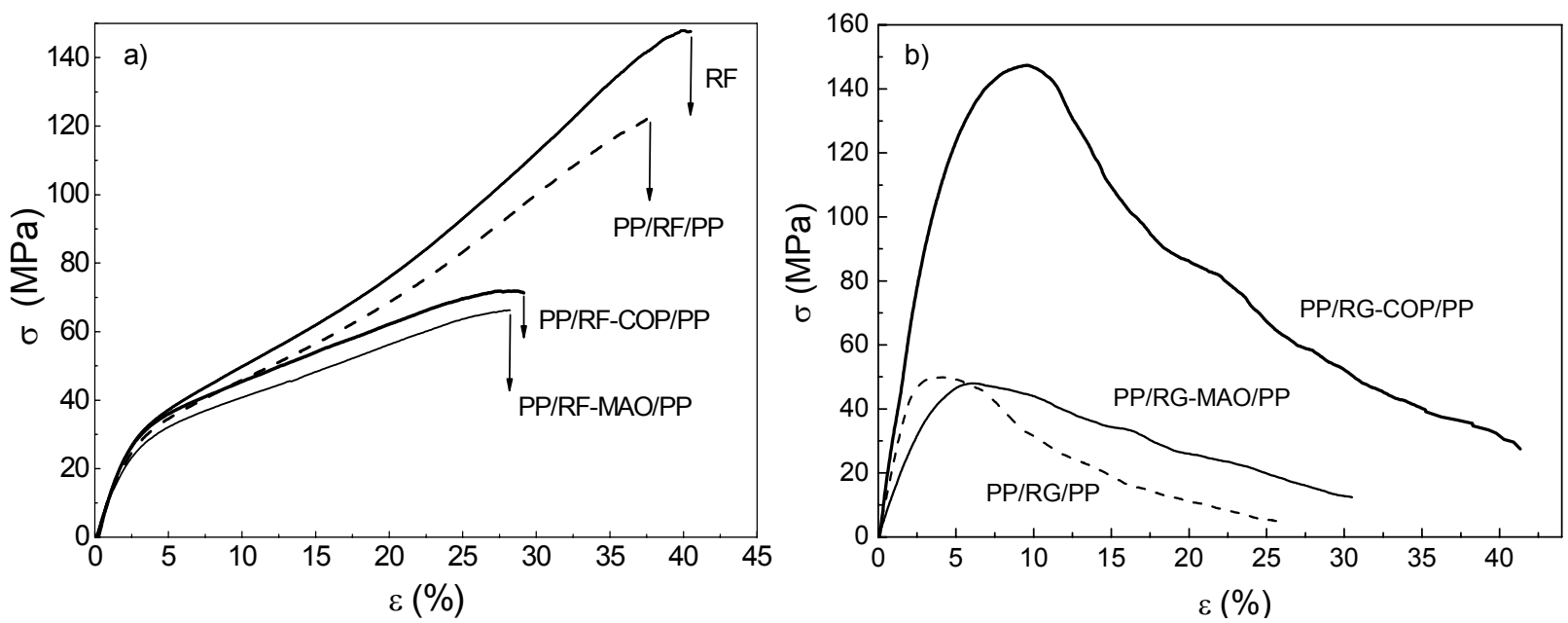

Fig. 4. Curvas tensión-deformación para los compuestos a) PP/RF/PP b) PP/RG/PP

Tabla 2. Propiedades obtenidas de las curvas tensión-deformación de todos los compuestos

\begin{tabular}{c|c|c|c}
\hline Compuesto & Módulo Elástico [MPa] & $\begin{array}{c}\text { Resistencia Mecánica } \\
{[\mathrm{MPa}]}\end{array}$ & $\begin{array}{c}\text { Elongación a Rotura } \\
{[\%]}\end{array}$ \\
\hline PP/RF/PP & $1189 \pm 147$ & $155 \pm 19,84$ & $40,71 \pm 8,3$ \\
\hline PP/RF-MAO/PP & $1130 \pm 88,66$ & $50,07 \pm 14,98$ & $30,12 \pm 4,7$ \\
\hline PP/RF-COP/PP & $1156 \pm 120$ & $60,91 \pm 7,51$ & $30,11 \pm 3,66$ \\
\hline \hline PP/RG/PP & $7803 \pm 1060$ & $48,0 \pm 4,3$ & $21,6 \pm 9,05$ \\
\hline PP/RG-MAO/PP & $9891 \pm 1029$ & $47,4 \pm 3,2$ & $25,6 \pm 3,5$ \\
\hline PP/RG-COP/PP & $9452 \pm 1017$ & $149,9 \pm 16,3$ & $40,05 \pm 6,3$ \\
\hline
\end{tabular}




\section{CONCLUSIONES}

Es este trabajo se analizó la influencia de la composición superficial de las fibras de vidrio cuando son usadas como base para la reacción de copolimerización de polipropileno sobre fibras de vidrio, medida a través de las propiedades mecánicas. La presencia de acrilatos claramente inhibe la formación del copolímero desde el vidrio. Siendo la reacción de copolimerización muy efectiva cuando se lleva a cabo directamente sobre el vidrio. Esto refuerza la idea de llevar a cabo la aplicación del proceso de polimerización in situ luego de la fabricación de las mismas, para un mayor aprovechamiento de los $\mathrm{OH}$ generados por la hidrólisis de la superficie del vidrio durante la formación de las fibras. También permite reducir los costos ya que sólo se llevaría a cabo la polimerización sin ningún tipo de recubrimiento intermedio.

\section{REFERENCIAS}

Arbelaiz, A., B. Fernandez, J.A Ramos, A. Retegi, R. Llano-Ponte, I. Mondragón, Mechanical properties of short flax fibre bundle/polypropylene composites: Influence of matrix/fibre modification, fibre content, water uptake and recycling, Composites Science and Technology, 65, 1582-1592 (2005).

Bledzki, A.K., J. Gassan, Composites reinforced with cellulose based fibres, Progress in Polymer Science, 24, 221-274, (1999).

DiBenedetto, A.T. Measurement of the thermomechanical stability of interphases by the embedded single fiber test Compos Sci Technol, 42(1-3), 103-123 (1991).

Etcheverry, M., D. Damiani, M.L Ferreira, S. Barbosa y N. Capiati, Chemical grafting of metallocene-catalyzed functional polypropylene copolymer on glass substrates through surface modification, J Appl Polym Sci: 109, 2815-2822 (2007).

Etcheverry, M., M.L. Ferreira, N.J. Capiati, A. Pegoretti, y S.E. Barbosa, Strengthening of polypropylene-glass fiber interface by direct metallocenic polymerization of propylene onto the fibers, Composites: Part A: 39,1915-1923 (2008).

Feller J.F. y Y. Grohens, Coupling ability of silane grafted poly(propene) at glass fibers/poly(propene) interface Composites: Part A: 35, 1-10 (2004).

Mallick P.K. Fiber Reinforced Composites, Ed. Faulkner L, Marcel Dekker, Chapter I, New York, USA (2003).

Monte, S. J. Titanate Coupling Agents, Functional fillers for plastics; by M. Xantos, 85-104 Wiley$\mathrm{VCH}$; Morlenbach, GE (2005).

Nielsen, L. y R. Landel, Mechanical Properties of Polymers and Composites, Ed. Marcel Dekker, Vol 2, New York (1994).

Xantos, M. Functional Fillers for Plastics, part II. Ed. M. Xantos, Molenbach, Germany: Wiley (2005). 